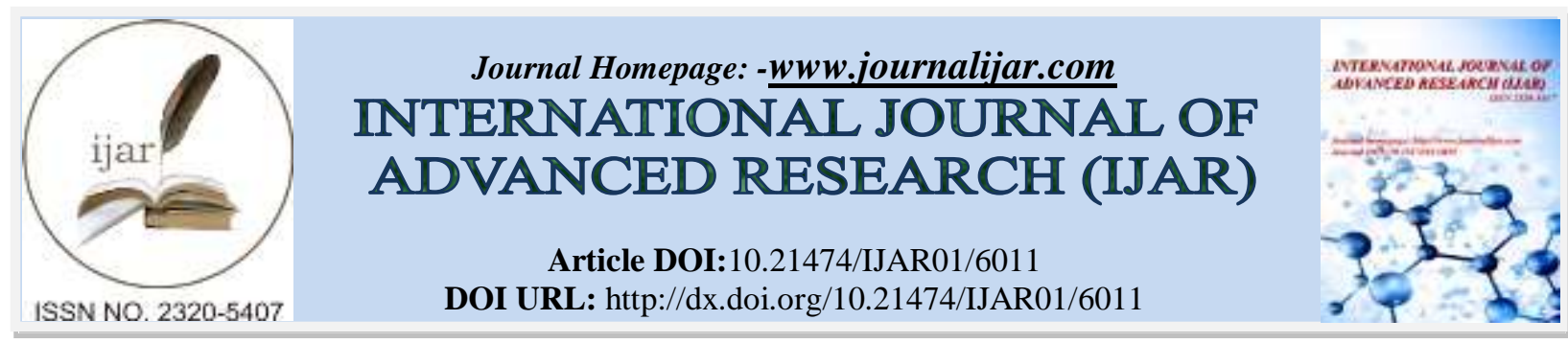

RESEARCH ARTICLE

\title{
DOES EVIDENCE SUPPORT PROSTHETIC OR SURGICAL REHABILITATION FOR PATIENTS WITH MAXILLARY DEFECT REGARDING THE QUALITY OF LIFE? SYSTEMATIC REVIEW.
}

\section{Sharaf .M.Y, DDS, MSc ${ }^{1}$, Ibrahim S.I , DDS, MSc, PhD ${ }^{2}$, Eskander A.E,dds, msc, phd ${ }^{3}$ and Shawky A F, DDS, MSc, $\mathrm{PhD}^{4}$.}

1. Assistant Lecture, Department of prosthodontics , Faculty of Dentistry, University of Beni-Suef. Egypt .

2. Professor,Department of prosthodontics, Faculty of oral and dental medicine, University of Cairo. Cairo ,Egypt.

3. Professor,Department of prosthodontics ,Faculty of oral and dental medicine,University of Cairo.Cairo ,Egypt.

4. Assistant professor, Department of prosthodontics , Faculty of Dentistry, University of Beni-Suef. Egypt .

\section{Manuscript Info}

\section{Manuscript History}

Received: 09 October 2017

Final Accepted: 11 November 2017

Published: December 2017

\section{Key words:-}

Obturator; Surgical Rehabilitation; Free Tissue Transfer; Maxillary Defects; Quality Of Life.

\begin{abstract}
Purpose: To assess the ability of prosthetic rehabilitation versus surgical rehabilitation in improving the QOL for patients with maxillary defects.

Material and methods: A systematic search of PubMed,Scopus data base, Cochrane database,Ovid database and Latin America \& Caribbean database for articles published before September 2017 was performed by two independent reviewers. A manual search of articles published from January 2000 to September 2017 was also conducted .Only English studies were included which evaluate the QoL in patients with head and neck cancers. Any confusion between the two independent reviewers was resolved by means of a moderated discussion between the reviewers.

Results: Five studies fulfilled the inclusion criteria for this study. Many parameters were used regarding evaluation of QOL as the EORTC Head and Neck 35 assessment, UW-QOL, OHIP-14 , VAS, OFS, MHI ,HAD,Body Satisfaction Scale,Oral symptom check list, Swallowing, Diet consist, Pain control,Postoertive complication and Speech . Two studies supported the surgical line of treatment for improving the QOL as compared to the prosthetic one another two studies showed a statistically insignificant improvement in the patients' QOL with the surgical line, while only one revealed insignificant difference in QoL with both lines of treatment.

Conclusions: Surgical rehabilitation provide a better line of treatment in improving the QOL for patients with maxillary defects, On the other hand prosthetic has proved effectiveness in the immediate postsurgical times as temporary strategy, and it has represented a good alternative when the surgical obturation is compromised .
\end{abstract}

Copy Right, IJAR, 2017,. All rights reserved. 


\section{Introduction:-}

Oral cancer is considered the 6th most frequent cancer representing about 1 to $3 \%$ of all human cancers. They may invade many different structures such as the oral cavity, oral pharynx, nasal cavity, nasal and laryngeal parts of the pharynx, paranasal sinuses, larynx, and salivary glands, ${ }^{1-3}$ where they have same clinical manifestations and management because of their adjacent anatomies. Resection of most malignant tumors can lead to defects resulting in oro-nasal communication. ${ }^{3-5}$

The maxilla is considered a vital and critical structure of the face, regarding the esthetics and function. It provides support for the maxillary teeth, orbital content, transmit the forces of mastication, separate the oral and nasal cavities, and provide attachment for the muscles of mastication and facial expression. ${ }^{6-9}$ As the maxilla is in close relationship with many critical and vital structures, the maxillary bone is usually included when resecting tumors that arise from the palate, nasal cavity, paranasal sinuses, orbital contents, overlying skin, or oral mucosa thus several adjacent structures are often resected in combination with the maxilla. ${ }^{7}$

Diagnosis of cancer and associated treatment consider a life altering and threating condition, causing fear and uncertainty of future. Thus head and neck cancer patient are at risk of financial, family ,social issues and increased emotional distress, as the life-saving surgical procedures often results in significant physical, physiological and psychological disabilities. $^{10-13}$

The resulting oro-nasal communication after resection leads to hypernasal speech, low speech intelligibility , nasal regurgitation of food and liquids due to incomplete separation between the nasal and oral caity, improper mastication, and disfigurement of facial apperence. Thus the social behavior and quality of life (QoL) will be adversely affected. ${ }^{14,15}$

Treatment planning for cancer patients has been improved in which postoperative quality of life factors are becoming increasingly important. ${ }^{16}$ Reconstruction can be achieved surgically with local, distant flaps, autologous pedicle flap, free tissue transfer, or vascularized bone composite tissue flaps, also prosthetic management may be carried out using different types of obturators or combinations of both line of treatment. ${ }^{8,17}$ Satisfactory levels of functionality and quality of life have been reported for both treatment alternatives. ${ }^{15,18,19}$ The size and the configuration of defect, residual bone, remaining dentition, soft tissue condition, and the patient's general health have to be considered regarding the selection of a suitable line of treatment. ${ }^{20-25}$

The application of dental implants has been found to be advantageous for the oral rehabilitation of patients who have undergone intraoral resections, as dental implants in bone grafts or free flaps after tumor resection was providing satisfactory results despite the thickness and mobility of soft tissues and scar contracture, as well as, the use of dental implants in the zygoma or in premaxilla improved obturator retention and stability. ${ }^{26-28}$

Quality of life is an important consideration in philosophy, medicine, religion, ecomomics and politics. In general, the term 'quality of life' is used to describe factors that influence the living conditions of a society or of the society's individuals. The WHO defines quality of life as 'the individual's perception of their position in life in the context of the culture and value systems inwhich they live and in relation to their goals, expectations, standards and concerns. ${ }^{29,30}$ Quality of life also includes physical health, personal circumstances, social relationships, functional activities and pursuits, as well as wider societal and economic influence.${ }^{31}$ For patients after maxillectomy, the psychological well-being and vitality are increasingly contributing to the evaluation of the therapeutic success, due to the high levels of psychological and physical trauma, ${ }^{32}$ Thus,optimal reconstruction of maxillectomy defects remains controversial. The decision whether to reconstruct or to obturator regarding the quality of life will be clarified.

\section{Materials and methods:-}

\section{Protocol and Eligibility Criteria:-}

The substructure of this systematic review is based on the PRISMA checklis and guidelines.${ }^{33,34}$ The focused question according to the PICO schema is: "which is better regarding the quality of life the prosthetic rehabilitation or surgical one in maxillectomy patients ?". This review question was based on recent clinical studies, including both prospective and retrospective, focused on the population, intervention,comparison, and result framework ${ }^{35}$ : population was defined as patients with maxillary defects; intervention was the method used to treat the maxillary 
defect; Comparison regarding the QOL for patients who had underwent prosthetic rehabilitation versus surgical one.The eligible studies should present the following characteristics: (1) randomized controlled trials, (2) prospective studies,(3) retrospective studies, (4) cohort studies, (5) published in English, (6) comparisons between prosthetic rehabilitation and surgical rehabilitation in the same study, .The exclusion criteria used were: (1) in vitro studies, (2) animal studies, (3) case series or case reports, (4) computer simulations, (5) patients or data repeated in other included articles.

\section{Search Strategy and Information Sources:-}

The systematic search for relevant literature was performed in the following database: PubMed, Scopus data base, Cochrane database, Ovid database and Latin America \& Caribbean database for articles published before may 2017 The following search terms were used regarding the quality of life ,prosthetic rehabilitation and surgical rehabilitation.:((()((()((()(Maxillary reconstructive surgical procedures) OR Maxillary reconstructive surgical procedures[MeSH Terms]) OR Maxillary surgery) OR Maxillary surgery[MeSH Terms]) OR Palatal reconstruction) OR Palatal reconstruction[MeSH Terms]) OR Maxillary surgical obturation) OR Maxillary graft) OR Surgical rehabilitation) OR Surgical management) OR Maxillary free flap) OR Maxillary microvascular free tissue transfers)) AND $(((()(()()()(()(((($ unilateral total maxillectomy) OR bilateral total maxillectomy) OR maxillary defects) OR maxillary tumor) OR maxillary cancer) OR maxillectomy) OR surgical Maxillary defect) OR acquired maxillary defect) OR maxillary defect)) OR obturator) OR obturators) OR maxillary obturator) OR maxillary obturators) OR palatal obturators) OR palatal obturator) OR palatal obturators[MeSH Terms]) OR obturator prostheses) OR maxillary obturation) OR obturation) OR Prosthetic rehabilitation) OR Prosthetic management)) AND ((Quality of life) OR Patient satisfaction).

Electronic search was accomplished by an iterative hand-search in the reference lists of the already identified articles. Endnote X7 was used for the electronic management of the literature.

The selection of the included papers were performed according to the eligibility criteria individually by two of the authors any disagreement between the independent reviewers was resolved by means of a moderated discussion between the reviewers .

\section{Results:-}

\section{Study selection:-}

A total of 1747 potentially relevant titles and abstracts were found by the electronic search and additional evaluation of reference lists. After removing 146 duplicate studies ,the remaining 1601 studies were screened,where 1579 publications were excluded based on the title and abstract, twenty two full-text articles were thoroughly evaluated, seventeen studies did not fulfilled the inclusion criteria of the present systematic review (Tab1). Finally the remaining five articles went into qualitative assessment (Fig. 1).

Because of the limited numbers of included studies, heterogenic study design, and incompletely reported data like, the quantitative data synthesis could not be performed in the way necessary for meta-analysis.

\section{Quality and risk of bias assessment:-}

A system modified from the US Agency for Healthcare Research and Quality Methods Guide for Comparative Effectiveness Reviews was used to assess the sources of possible bias. As it's unwise to use the Cochrane collaboration tool for assessing risk of bias for randomized controlled trials since all included studies were not randomized.The criteria were judged with high, medium, low, or unknown risk of bias: case selection bias and confounders, attrition bias, detection bias, and reporting bias, and a summary of the risk. ${ }^{36}$ (Tab 2 )

Of the five selected studies one were prospective, two were retrospective and two were cross sectional study .From the five included articles, a total of 167 patients ( 97 male ,70 female),the age of the patients ranged from 16 to 91 years in the selected studies. Of all of the patients included in this study, 64 patients $(38.32 \%)$ were rehabilitated with obturator prostheses and 103 patients (61.68\%) were treated with surgical obturation. One study reported that patients who underwent surgical rehabilitation were rehabilitated with intermediate temporary obturator only 11 patient of 47 patients. ${ }^{51}$ Various tools and questionnaires were used for measuring QOL as the EORTC Head and Neck 35 assessment ,the University of Washington Head and Neck Questionnaire (UW-QOL), the Oral Health Impact Profile -14 (OHIP-14) , Visual Analogue Scale (VAS), the Obturator Functioning Scale (OFS), Mental 
Health Inventory (MHI) ,Hospital Anxiety Depression (HAD),Body Satisfaction Scale,Oral symptom check list, Swallowing, Diet consist, Pain control, Postoertive complication and Speech. ${ }^{32,49-52}$ Tab 3.

Regarding the defect classifications, one of the selected studies used Aramany classification, ${ }^{49}$ one used Brown classification system of defects, ${ }^{50}$ one used Okay et al classification, ${ }^{32}$ one used new classification ${ }^{51,53}$ and one study described only the extension of the surgical resection. ${ }^{52}$ The most common defect type was IIa-b Brown classification and class II okay classification. ${ }^{32,49-52}$ One study reported that the size of the defect affects significantly the difference between QOL scores for both lines of treatment, ${ }^{50}$ as well as, other study reported that the size and shape of the defect, especially in patients who receive radiation therapy, represent one of the most significant negative points. ${ }^{51}$ Where one study reported that postoperative radiotherapy affects the functioning of obturator prostheses. 49

Regarding the patients' tumors, the most frequently diagnosed type of cancer was squamous cell carcinoma, followed by mucoepidermoid carcinoma and rare variants of malignant neoplasms of palate minor salivary glands. ${ }^{32,49-52}$ Only one study reported the stage of the tumor for the patients evaluated as stage IV followed by stage III as the most common stages. ${ }^{52}$ Only one study evaluated the dental status of selected patients ${ }^{32}$, ultimately, all the studies were done at five medical centers. However,only two studies were done in the same country. ${ }^{32,49-52}$

No meta-analyses were performed because of the differences in the questionnaires, the variations in the ways in which the results were presented, and the differing methodologies of the included studies.

Two studies reported that surgical line can achieve superior function and QOL outcomes. ${ }^{49,51}$, where the other two studies reported that the surgical rehabilitation showed better results regarding the QOL but the difference was statistically insignificant $^{32,50}$ while only one revealed no significant difference in QoL with both lines of treatment. ${ }^{52}$

Table 1:- The excluded studies.

\begin{tabular}{|c|c|c|}
\hline & Author ,title and year & Cause of exclusion \\
\hline 1 & $\begin{array}{l}\text { Rieger et al,Surgical reconstruction versus prosthetic obturation } \\
\text { of extensive soft palate defects: a comparison of speech outcomes } \\
.2009^{37}\end{array}$ & Did not specifically evaluate QoL \\
\hline 2 & $\begin{array}{l}\text { Borlasse et al,Use of obturators in rehabilitation of maxillectomy } \\
\text { defects. } 2000^{38}\end{array}$ & Did not specifically evaluate QoL \\
\hline 3 & $\begin{array}{l}\text { Rieger et al, maxillary obturators: the relationship between } \\
\text { patient satisfaction and speech outcome } .2003^{39}\end{array}$ & Did not specifically evaluate QoL \\
\hline 4 & $\begin{array}{l}\text { Moreno et al, Microvascular free flap reconstruction versus } \\
\text { palatal obturation for maxillectomy defects } .2009^{40}\end{array}$ & Did not specifically evaluate QoL \\
\hline 5 & $\begin{array}{l}\text { Lethaus et al, Surgical and prosthetic reconsiderations in patients } \\
\text { with } \\
\text { Maxillectomy } .2010^{41}\end{array}$ & $\begin{array}{l}\text { Only one } \\
\begin{array}{l}\text { homogenous } \\
\text { comparisons } \\
\text { obturator) }\end{array}\end{array}$ \\
\hline 6 & $\begin{array}{l}\text { Rieger et al, Comparison of Speech and Aesthetic Outcomes in } \\
\text { Patients } \\
\text { with Maxillary Reconstruction versus Maxillary Obturators after } \\
\text { Maxillectomy, } 2011^{18}\end{array}$ & Did not specifically evaluate QoL \\
\hline 7 & $\begin{array}{l}\text { Hertramp et al } \quad \text { Quality of life of patients } \\
\text { with maxillofacial defects after treatment for malignancy,2004 }\end{array}$ & $\begin{array}{l}\text { The control group is not malignant } \\
\text { patient }\end{array}$ \\
\hline 8 & $\begin{array}{l}\text { Kornblith et al quality of life of maxillectomy patients using an } \\
\text { obturator prosthesis } .1996^{19}\end{array}$ & $\begin{array}{l}\text { Only one homogenous group no } \\
\text { comparisons (obturator only) }\end{array}$ \\
\hline 9 & $\begin{array}{l}\text { Brown et al,A modified classification for the maxillectomy } \\
\text { Defect } .2000^{5}\end{array}$ & $\begin{array}{l}\text { As both groups were divided according } \\
\text { to the classification of defects not the } \\
\text { intervention. }\end{array}$ \\
\hline 10 & $\begin{array}{l}\text { Irish et al, Quality of life in patients with maxillectomy } \\
\text { prostheses.2009. } 15\end{array}$ & $\begin{array}{l}\text { Only one homogenous group no } \\
\text { comparisons (obturator only) }\end{array}$ \\
\hline 11 & $\begin{array}{l}\text { Depprich et al,Evaluation of quality of life of patients with } \\
\text { maxillofacial defects after } \\
\text { prosthodontic therapy with obturator prostheses }, 2010^{14}\end{array}$ & $\begin{array}{l}\text { Only one homogenous group no } \\
\text { comparisons (obturator only) }\end{array}$ \\
\hline
\end{tabular}




\begin{tabular}{|c|c|c|}
\hline 12 & $\begin{array}{l}\text { Riaz\&Warriach } \\
\text { prostheses } 2010^{43}\end{array}$ & $\begin{array}{l}\text { Only one homogenous group no } \\
\text { comparisons (obturator only) }\end{array}$ \\
\hline 13 & $\begin{array}{l}\text { Kreeft et al.Oral function after Maxillectomy and reconstruction } \\
\text { with obturator }, 2012 .{ }^{44}\end{array}$ & $\begin{array}{l}\text { Only one homogenous group no } \\
\text { comparisons (obturator only) }\end{array}$ \\
\hline 14 & $\begin{array}{l}\text { Chigurupati et al .Quality of life after maxillectomy and } \\
\text { prosthetic obturator rehabilitation, } 2013^{45}\end{array}$ & $\begin{array}{l}\text { Only one homogenous group no } \\
\text { comparisons (obturator only) }\end{array}$ \\
\hline 15 & $\begin{array}{l}\text { Kumar et al.Assessment of the quality of } \\
\text { life in maxillectomy patients: a longitudinal } \\
\text { study } 2013^{46}\end{array}$ & $\begin{array}{l}\text { Only one homogenous group no } \\
\text { comparisons (obturator only) }\end{array}$ \\
\hline 16 & $\begin{array}{l}\text { Said et al ,Masticatory function and oral health-related quality of } \\
\text { life in patients after partial maxillectomies with closed or open } \\
\text { defects. } 2016^{47}\end{array}$ & $\begin{array}{l}\text { Because the closed defect related to } \\
\text { healing not surgical intervention }\end{array}$ \\
\hline 17 & $\begin{array}{l}\text { Costa } \mathrm{H} \text { et al .Microsurgical Reconstruction Of The Maxilla - } \\
\text { Algorithm And Concepts. } 2015^{48}\end{array}$ & $\begin{array}{l}\text { Only one homogenous group no } \\
\text { comparisons (surgical group) }\end{array}$ \\
\hline
\end{tabular}

Table 2:- Quality and risk of bias assessment

\begin{tabular}{|c|c|c|c|c|c|c|c|c|}
\hline Author & Year & Study type & $\begin{array}{l}\text { Selection } \\
\text { bias } \\
\text { (homogeneit } \\
\text { y } \\
\text { and } \\
\text { confounders) }\end{array}$ & $\begin{array}{l}\text { Performanc } \\
\text { e } \\
\text { bias } \\
\text { (fidelity } \\
\text { to protocol) }\end{array}$ & $\begin{array}{l}\text { Attrition } \\
\text { bias } \\
\text { (loss of } \\
\text { participants } \\
\text { ) }\end{array}$ & $\begin{array}{l}\text { Detection } \\
\text { bias } \\
\text { (reliable } \\
\text { measures } \\
\text { ) }\end{array}$ & $\begin{array}{l}\text { Reportin } \\
\text { g bias } \\
\text { (selective } \\
\text { reporting } \\
\text { or } \\
\text { conflict } \\
\text { interests) }\end{array}$ & $\begin{array}{l}\text { Summary } \\
\text { assessmen } \\
t \\
\text { risk of } \\
\text { bias }\end{array}$ \\
\hline $\begin{array}{l}\text { Genden } \\
\text { et } \mathrm{al}^{49}\end{array}$ & $\begin{array}{l}200 \\
3\end{array}$ & Prospective & $\mathrm{H}$ & $\mathrm{L}$ & $\mathrm{L}$ & $\mathrm{L}$ & $\mathrm{L}$ & $\mathrm{L}$ \\
\hline $\begin{array}{l}\text { Rogers } \\
\text { et } \mathrm{al}^{50}\end{array}$ & $\begin{array}{l}200 \\
3 \\
\end{array}$ & Cross section & $\mathrm{H}$ & $\mathrm{L}$ & $\mathrm{L}$ & $\mathrm{L}$ & $\mathrm{L}$ & $\mathrm{L}$ \\
\hline $\begin{array}{l}\text { Wang } \\
\text { et } \mathrm{al}^{32}\end{array}$ & $\begin{array}{l}201 \\
6\end{array}$ & Cross section & $\mathrm{H}$ & $\mathrm{L}$ & $\mathrm{L}$ & $\mathrm{L}$ & $\mathrm{L}$ & $\mathrm{L}$ \\
\hline $\begin{array}{l}\text { Giorgio } \\
\text { Pompa, } \\
\text { et al }{ }^{51}\end{array}$ & $\begin{array}{l}201 \\
7\end{array}$ & $\begin{array}{l}\text { Retrospectiv } \\
\mathrm{e}\end{array}$ & $\mathrm{H}$ & $\mathrm{H}$ & $\mathrm{L}$ & $\mathrm{L}$ & $\mathrm{M}$ & $\mathrm{M}$ \\
\hline $\begin{array}{l}\text { Murph } \\
\text { y et } \mathrm{al}^{52}\end{array}$ & $\begin{array}{l}201 \\
2\end{array}$ & $\begin{array}{l}\text { Retrospectiv } \\
\mathrm{e}\end{array}$ & $\mathrm{H}$ & $\mathrm{L}$ & $\mathrm{M}$ & $\mathrm{L}$ & $\mathrm{L}$ & $\mathrm{M}$ \\
\hline
\end{tabular}

Table 3:- The characteristics of the included studies

\begin{tabular}{|c|c|c|c|c|c|c|c|}
\hline & $\begin{array}{l}\text { Author (Year) } \\
\text { Journal }\end{array}$ & Title & $\begin{array}{l}\text { Study } \\
\text { Design } \\
\text { (Follow-up } \\
\text { Period in } \\
\text { Years) }\end{array}$ & $\begin{array}{l}\text { Patient } \\
\text { Informati } \\
\text { on }\end{array}$ & $\begin{array}{l}\text { Questionnair } \\
\text { es } \\
\text { for QoL }\end{array}$ & The Results & $\begin{array}{l}\text { No. of } \\
\text { Patients } \\
\text { and Class } \\
\text { of Defects }\end{array}$ \\
\hline 1 & $\begin{array}{l}\text { Genden et al } \\
(2003) \\
\text { Archives of } \\
\text { Otolaryngolog } \\
\text { y-Head \& } \\
\text { Neck } \\
\text { Surgery }\end{array}$ & $\begin{array}{l}\text { Comparison } \\
\text { of } \\
\text { functional } \\
\text { and } \\
\text { quality-of- } \\
\text { life } \\
\text { outcomes in } \\
\text { patients with } \\
\text { and without } \\
\text { palatomaxill } \\
\text { ary } \\
\text { reconstructio } \\
\text { n: } \\
\text { a preliminary }\end{array}$ & Prospective & $\begin{array}{l}\mathrm{N}=8 \quad(5 \\
\text { male, } 3 \\
\text { female); } \\
\text { The range } \\
\text { of } \\
\text { age=18- } \\
69 \text { years; } \\
4 \text { OB } \\
4 \text { SR }\end{array}$ & $\begin{array}{l}\text { 1)Chewing } \\
\text { performance } \\
\text { 2)Swallowin } \\
\text { g } \\
\text { Quality of } \\
\text { Life } \\
\text { Survey } \\
\text { (SWALQO } \\
\text { L) } \\
\text { 3)Subjective } \\
\text { speech } \\
\text { perception, } \\
\text { 4)Nasometr }\end{array}$ & $\begin{array}{l}\text { (Rehabilitation of } \\
\text { the } \\
\text { hemipaltomaxillect } \\
\text { omy with } \\
\text { vascularized bone- } \\
\text { containing free } \\
\text { flaps defect can } \\
\text { achieve superior } \\
\text { function and QOL } \\
\text { outcomes relative to } \\
\text { defect matched } \\
\text { patient with a } \\
\text { prosthetic } \\
\text { obturator.) }\end{array}$ & $\begin{array}{l}\text { Class II } \\
\text { Aramany } \\
\text { classificatio } \\
\mathrm{n}\end{array}$ \\
\hline
\end{tabular}




\begin{tabular}{|c|c|c|c|c|c|c|c|}
\hline & & report. & & & $\begin{array}{l}\text { y, } \\
\text { 5)Doner site } \\
\text { assessement, } \\
\text { Disabilities } \\
\text { of the Arm, } \\
\text { Shoulder } \\
\text { and Hand } \\
\text { (DASH) } \\
\text { 6) American } \\
\text { Association } \\
\text { of } \\
\text { Orthopedic } \\
\text { Surgeons } \\
\text { (AAOS) } \\
\text { Hip and } \\
\text { Knee }\end{array}$ & $\begin{array}{l}\text {-SWAL-QOL } \\
\text { Subjective speech } \\
\text { perception showed } \\
\text { higher QoL for } \\
\text { SG than OB } \\
\text {-Donor site } \\
\text { questionnaires } \\
\text { showed same level } \\
\text { of quality of life } \\
\text {-Chewing } \\
\text { performance, are } \\
\text { Nasometry higher in the SG but } \\
\text { not significant }\end{array}$ & \\
\hline 2 & $\begin{array}{l}\begin{array}{l}\text { Rogers et al } \\
(2003) \\
\text { Journal of } \\
\text { Oral } \\
\text { Maxillofacial } \\
\text { Surgery }^{50}\end{array} \\
\end{array}$ & $\begin{array}{l}\text { Health- } \\
\text { related } \\
\text { quality of } \\
\text { life } \\
\text { after } \\
\text { maxillectom } \\
\text { y: a } \\
\text { comparison } \\
\text { between } \\
\text { prosthetic } \\
\text { obturation } \\
\text { and free flap }\end{array}$ & $\begin{array}{l}\text { Cross } \\
\text { sectional } \\
\text { study }\end{array}$ & $\begin{array}{l}\mathrm{N}=28(18 \\
\text { male, } \\
10 \\
\text { female); } \\
\text { mean } \\
\text { age=64 } \\
\text { years; } \\
10 \mathrm{OB} \\
18 \mathrm{SR}\end{array}$ & $\begin{array}{l}\text { 1)the } \\
\text { University } \\
\text { of } \\
\text { Washington } \\
\text { Head and } \\
\text { Neck } \\
\text { Questionnair } \\
\text { e UW-QOL } \\
\text { 2)European } \\
\text { Organizatio } \\
\text { n for } \\
\text { Research } \\
\text { and } \\
\text { Treatment } \\
\text { of } \\
\text { Cancer Core } \\
\text { QOL } \\
\text { Questionnair } \\
\text { e } \\
\text { (EORTC } \\
\text { QLQ C30) } \\
\text { 3)-EORTC } \\
\text { Head and } \\
\text { Neck } \\
\text { (EORTC } \\
\text { QLQ } \\
\text { H\&N35) } \\
\text { 4)Hospital } \\
\text { Anxiety } \\
\text { Depression } \\
\text { (HAD) } \\
\text { 5)Body } \\
\text { Satisfaction } \\
\text { Scale, } \\
\text { 6)Oral } \\
\text { symptom } \\
\text { check list, }\end{array}$ & $\begin{array}{l}\text { OB group indicate } \\
\text { more problems with } \\
\text { appearance in } \\
\text { UWQOL. } \\
\text { Anxiety scores were } \\
\text { slightly higher in } \\
\text { OB group but not } \\
\text { significant . } \\
\text { OB group is aware } \\
\text { of their upper teeth, } \\
\text { more self- } \\
\text { conscious, less } \\
\text { satisfied with upper } \\
\text { dentures, and } \\
\text { less satisfied with } \\
\text { function } \\
\text { No statistically } \\
\text { significant } \\
\text { differences were } \\
\text { identified between } \\
\text { OB } \\
\text { SRHowever, size of } \\
\text { surgical defect } \\
\text { affected "activity" } \\
\text { and "recreation" } \\
\text { domains } \\
\text { UWQOL, of } \\
\text { and "physical } \\
\text { functioning" and } \\
\text { "quality of life" } \\
\text { domains } \\
\text { EORTC. of }\end{array}$ & $\begin{array}{l}\text { OB } \\
\text { 1-I } \\
\text { 7-IIa } \\
\text { 1-IIb } \\
\text { 1-IIIb } \\
\text { SR } \\
\text { 1-I } \\
6 \text {-IIa } \\
\text { 5-IIb } \\
\text { 1-IIIa } \\
\text { 1-IIIb } \\
\text { 2-Iva } \\
\text { 1-IVb } \\
\text { Brown } \\
\text { classificatio } \\
\text { n }\end{array}$ \\
\hline 3 & \begin{tabular}{|lr} 
Wang & et \\
al(2016) & \\
Clin. & Oral
\end{tabular} & $\begin{array}{l}\text { Functional } \\
\text { outcome and } \\
\text { quality of }\end{array}$ & $\begin{array}{l}\text { Cross } \\
\text { sectional } \\
\text { study }\end{array}$ & $\begin{array}{l}\mathrm{N}=38 \quad(23 \\
\text { male, } \\
15\end{array}$ & $\begin{array}{l}\text { 1)the } \\
\text { Obturator } \\
\text { Functioning }\end{array}$ & $\begin{array}{ll}\text { The patient's } & \text { QOl } \\
\text { with } & \text { both } \\
\text { approaches } & \text { was }\end{array}$ & $\begin{array}{l}15 \text { Class Ib } \\
15 \text { Class II } \\
8 \text { Class III }\end{array}$ \\
\hline
\end{tabular}




\begin{tabular}{|c|c|c|c|c|c|c|c|}
\hline & Impl. Res. ${ }^{32}$ & $\begin{array}{l}\text { life } \\
\text { after a } \\
\text { maxillectom } \\
\text { y: a } \\
\text { comparison } \\
\text { between an } \\
\text { implant } \\
\text { supported } \\
\text { obturator and } \\
\text { implant } \\
\text { supported } \\
\text { fixed fred } \\
\text { prostheses in } \\
\text { a free } \\
\text { vascularized } \\
\text { flap }\end{array}$ & & $\begin{array}{l}\text { female); } \\
\text { The range } \\
\text { of } \\
\text { age=20- } \\
70 \text { years; } \\
\text {; } \\
18 \text { OB } \\
20 \text { SR }\end{array}$ & $\begin{array}{l}\text { Scale (OFS), } \\
\text { 2)EORTC } \\
\text { Head and } \\
\text { Neck } 35 \\
\text { assessment } \\
\text { 3) Mental } \\
\text { Health } \\
\text { Inventory } \\
\text { (MHI) }\end{array}$ & 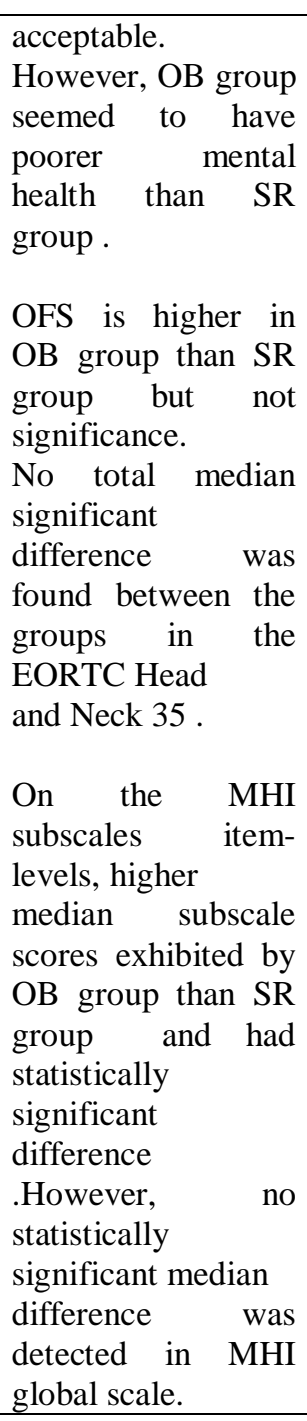 & $\begin{array}{l}\text { Okay et al } \\
\text { classificatio } \\
\mathrm{n}\end{array}$ \\
\hline 4 & $\begin{array}{l}\text { Giorgio } \\
\text { Pompa, et al } \\
2017 \\
\text { Journal of } \\
\text { International } \\
\text { Dental and } \\
\text { Medical } \\
\text { Research }^{51}\end{array}$ & $\begin{array}{l}\text { Quality of } \\
\text { Life in } \\
\text { Patients } \\
\text { Rehabilitated } \\
\text { with Palatal } \\
\text { Obturator } \\
\text { without } \\
\text { Reconstructi } \\
\text { on Versus } \\
\text { Fixed } \\
\text { Implant- } \\
\text { Prosthesis } \\
\text { after Reconstructi } \\
\text { on of } \\
\text { Maxillectom } \\
\text { y Defects }\end{array}$ & $\begin{array}{l}\text { Retrospecti } \\
\text { ve }\end{array}$ & $\begin{array}{l}\mathrm{N}=68 \text { (39 } \\
\text { male, } \\
29 \\
\text { female); } \\
\text { The range } \\
\text { of } \\
\text { age=16- } \\
75 \text { years; } \\
21 \text { OB } \\
47 \mathrm{SR}\end{array}$ & $\begin{array}{l}\text { 1)Visual } \\
\text { Analogue } \\
\text { Scale (VAS) } \\
\text { regarding } \\
\text { the OB only } \\
\text { 2) the Oral } \\
\text { Health } \\
\text { Impact } \\
\text { Profile -14 } \\
\text { (OHIP-14) }\end{array}$ & $\begin{array}{l}\text { Patients in SR } \\
\text { group resume their } \\
\text { normal daily } \\
\text { activities and work } \\
\text { life earlier than } \\
\text { those ones in OB } \\
\text { group, they recover } \\
\text { more successfully } \\
\text { their oral function } \\
\text { (chewing, } \\
\text { swallowing, } \\
\text { speech), they are } \\
\text { completely } \\
\text { reintegrated in their } \\
\text { social dimension } \\
\text { and show higher } \\
\text { satisfaction for } \\
\text { rehabilitation. }\end{array}$ & \begin{tabular}{ll}
\multicolumn{2}{l}{ SR } \\
9 & $\mathrm{U} 1-\mathrm{R}$ \\
4 & $\mathrm{U} 1+2-\mathrm{R}$ \\
7 & $\mathrm{U} 2-\mathrm{R}$ \\
12 & $\mathrm{U} 3-\mathrm{R}$ \\
2 & $\mathrm{U} 4-\mathrm{R}$ \\
7 & $\mathrm{UC2}-\mathrm{R}$ \\
4 & $\mathrm{UC3}-\mathrm{R}$ \\
2 & $\mathrm{UC} 4-\mathrm{R}$ \\
OB \\
$4 \mathrm{U} 1-\mathrm{C}$ \\
3 & $\mathrm{U} 1+2-\mathrm{C}$ \\
5 & $\mathrm{U} 2-\mathrm{C}$ \\
9 & $\mathrm{U} 3-\mathrm{C}$ \\
New \\
classificatio \\
$\mathrm{n}^{54}$
\end{tabular} \\
\hline 5 & $\begin{array}{lr}\text { Murphy } & \text { et } \\
\text { al,2015 } & \text { J } \\
\text { Oral } & \\
\end{array}$ & $\begin{array}{l}\text { Quality of } \\
\text { Life Factors } \\
\text { and Survival }\end{array}$ & $\begin{array}{l}\text { Retrospecti } \\
\text { ve }\end{array}$ & $\begin{array}{l}\mathrm{N}=25 \quad(12 \\
\text { male, } \\
13\end{array}$ & $\begin{array}{l}\text { 1)Swallowin } \\
\text { g } \\
\text { 2)Diet }\end{array}$ & $\begin{array}{l}\text { Obturator } \\
\text { placement } \\
\text { was found to be an }\end{array}$ & $\begin{array}{l}\text { Total and } \\
\text { extended } \\
\text { maxillectom }\end{array}$ \\
\hline
\end{tabular}




\begin{tabular}{|c|c|c|c|c|c|}
\hline $\begin{array}{l}\text { MaxillofacSur } \\
\mathrm{g}^{52}\end{array}$ & $\begin{array}{l}\text { After } \\
\text { Total or } \\
\text { Extended } \\
\text { Maxillectom } \\
\text { y for } \\
\text { Sinonasal } \\
\text { Malignancies }\end{array}$ & $\begin{array}{l}\text { female); } \\
\text { The range } \\
\text { of } \\
\text { age=47- } \\
91 \text { years; } \\
11 \text { OB } \\
14 \text { SR }\end{array}$ & $\begin{array}{l}\text { consist } \\
\text { 3)Pain } \\
\text { control } \\
\text { 4)Postoertiv } \\
\mathrm{e} \\
\text { complicatio } \\
\mathrm{n} \\
\text { 5)Speech } \\
\text { 6)Survival }\end{array}$ & $\begin{array}{l}\text { equivalent } \\
\text { reconstructive } \\
\text { option } \\
\text { with respect to the } \\
\text { quality of life } \\
\text { factors and } \\
\text { complications } \\
\text { in these patients. }\end{array}$ & $\mathrm{y}$ \\
\hline
\end{tabular}

\begin{tabular}{|c|c|c|c|c|c|c|}
\hline & $\begin{array}{l}\text { Dental } \\
\text { Status }(\%)\end{array}$ & $\begin{array}{l}\text { No. of Patients } \\
\text { and Disease } \\
\text { Type }\end{array}$ & $\begin{array}{l}\text { Adjuvant } \\
\text { Treatments }\end{array}$ & $\begin{array}{l}\text { Implant } \\
\text { Therapy } \\
\text { (Yes/No) }\end{array}$ & $\begin{array}{l}\text { Geographic } \\
\text { Location of } \\
\text { Patients } \\
\text { (Country) }\end{array}$ & $\begin{array}{l}\text { Medical } \\
\text { Centers }\end{array}$ \\
\hline 1 & NR & $\begin{array}{l}1 \text { AdCC } \\
2 \text { Osteosarcoma } \\
2 \text { SCC } \\
2 \text { Ameloblastoma } \\
1 \text { Cylindric cell carcinoma }\end{array}$ & $\begin{array}{l}4 \mathrm{RT} \\
(2 \mathrm{OB}, 2 \\
\mathrm{SR}) \\
2 \mathrm{CT} \\
(1 \quad \mathrm{OB}, 1 \\
\mathrm{SR})\end{array}$ & No & USA & $\begin{array}{l}\text { Department of } \\
\text { Otolaryngology } \\
\text { - Head and Neck } \\
\text { Surgery }\end{array}$ \\
\hline 2 & NR & NR & $\begin{array}{l}4 \mathrm{RT} \\
(1 \mathrm{OB}, 3 \\
\mathrm{SR})\end{array}$ & Yes & $\begin{array}{l}\text { United } \\
\text { Kingdom. }\end{array}$ & $\begin{array}{l}\text { Regional } \\
\text { Maxillofacial Unit, } \\
\text { University } \\
\text { Hospital Aintree, } \\
\text { Aintree } \quad \text { Trust, } \\
\text { Liverpool }\end{array}$ \\
\hline 3 & $\begin{array}{l}29 \text { Partially } \\
\text { Edentulous } \\
9 \\
\text { Edentulous }\end{array}$ & $\begin{array}{l}8 \text { Gingival squamous cell } \\
\text { carcinoma } \\
7 \text { Ameloblastoma } \\
5 \text { Maxilla sinus carcinoma } \\
6 \text { Hemangioma } \\
6 \text { Nasopharyngeal carcinoma } \\
2 \text { Palate mixed tumor } \\
4 \text { Other }\end{array}$ & $\begin{array}{l}11 \mathrm{RT} \\
(5 \mathrm{OB}, 6 \\
\text { SR) }\end{array}$ & Yes & China & $\begin{array}{l}\text { Department of Oral } \\
\text { and Maxillofacial } \\
\text { Surgery Oral } \\
\text { and the Department } \\
\text { of Implantology, } \\
\text { Shanghai Ninth } \\
\text { People's Hospital } \\
\text { affiliated } \\
\text { with Shanghai Jiao } \\
\text { Tong University, } \\
\text { School } \\
\text { of Medicine } \\
\text { Hospital Aintree }\end{array}$ \\
\hline 4 & NR & $\begin{array}{l}2 \text { Ameloblastoma } \\
1 \\
\text { keratocysticodontogenictumor } \\
1 \text { pleomorphic adenoma } \\
49 \text { squamous cell carcinomas } \\
12 \text { mucoepidermoidcarcinoma } \\
\text { and rare variants of malignant } \\
\text { neoplasms of palate minor } \\
\text { salivary glands } \\
1 \text { ameloblastic carcinoma, } \\
1 \text { chondroblastic osteosarcoma } \\
1 \text { rhabdomyosarcoma }\end{array}$ & $\begin{array}{l}38 \mathrm{RT} \\
17 \mathrm{CT} \\
\mathrm{RT}\end{array}$ & Yes & Italy. & $\begin{array}{l}\text { Department of } \\
\text { Odontostomatologic } \\
\text { and Maxillofacial } \\
\text { Sciences Sapienza } \\
\text { University of Rome }\end{array}$ \\
\hline 5 & $\mathrm{Nr}$ & $\begin{array}{l}13 \text { Squamous cell carcinoma. } \\
1 \text { Adenocarcinoma. } \\
4 \text { Adenoid cystic carcinoma. }\end{array}$ & $\begin{array}{l}6 \mathrm{RT} \\
4 \mathrm{CT} \\
7 \mathrm{RT} \& \mathrm{CT} \\
\end{array}$ & YES & USA & $\begin{array}{l}\text { University } r \text { of } \\
\text { Maryland Marlene } \\
\text { and Stewart }\end{array}$ \\
\hline
\end{tabular}




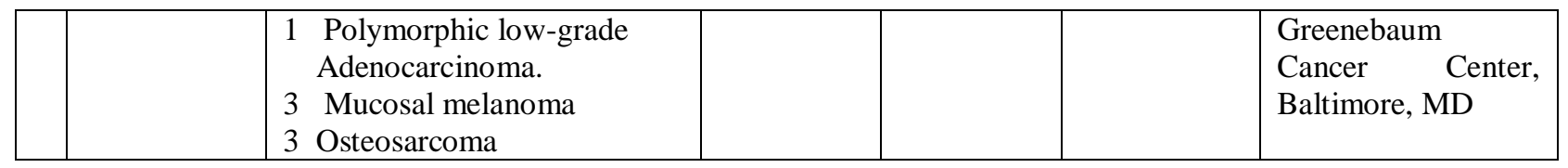

$\mathrm{N}$ number ,OB obturator, SR surgical rehabilitation ,NR not reported ,RT radiotherapy ,CT chemotherapy

Fig 1:-Prisma Flowchart of search strategy

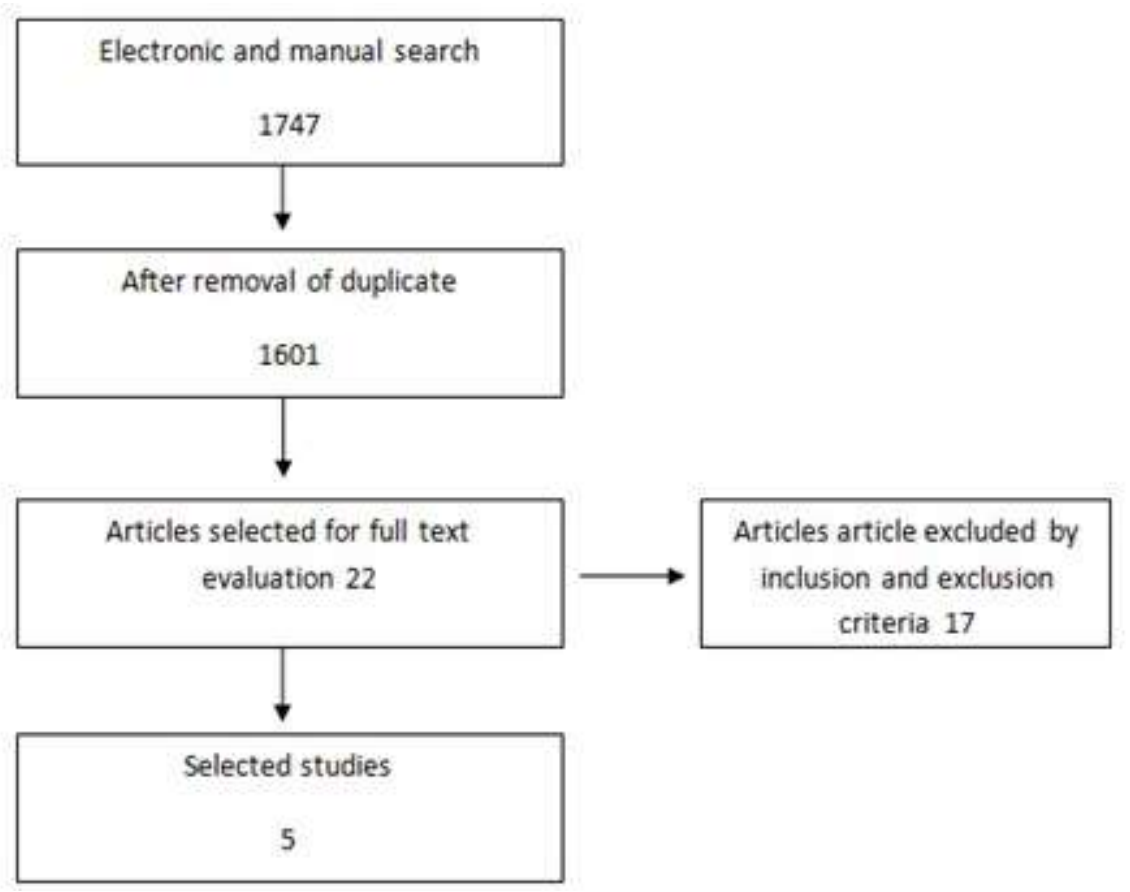

\section{Discussion:-}

This systematic review revealed a small number of studies that evaluated the QoL of patients with maxillary defects who underwent prosthetic rehabilitation or surgical rehabilitation. None of the included studies were randomized clinical trials that revealed a well-designed methodology for clinical studies as there is no blinding, randomization, concealment and sample size calculation. ${ }^{54}$

The patients' age could be considered an influencing factor for selection of the type of rehabilitation. ${ }^{55,56}$ Two studies were dealing with patients of average age 64 and 67.8 years. ${ }^{50.52}$ While in the remaining three studies younger age were included as the age ranged from second to seventh decades, ${ }^{32,49-52}$ so all studies included all variation of age. The size of the defects play a great role regarding QOL as larger defects are more difficult to fit with obturator prosthesis because the prosthesis is heavier. Thereby potentially creating problems related to leakage of fluid and food and causing hypernasal speech ${ }^{37}$ Similarly, the success of the free tissue transfers also depends upon the size of the defects. ${ }^{57}$ Defects that are larger than one third of the hard palate are challenging to be surgically reconstructed using local flaps and may not be completely closed in a single step. ${ }^{38,57}$ In the included studies two studies made matching of the defect and equivalent distribution of the type of the defects between both groups. ${ }^{32,49}$ while in other studies the more larger defects were reconstructed surgically rather than prosthetically with obturator. ${ }^{50,52}$ Another important factor for better obturator function were the extent of soft palate resection, that is to say one third or less is better regarding Qol, According to the included studies, one study deals with soft palate defects and reported an insignificant difference between both groups. ${ }^{50}$ In this systematic review, the majority of the defects were type IIa-b Brown classification and class II okay classification, which reveals that most patients had their alveolar bone and the walls of maxillary sinus resected without involvement of the orbit. Additionally, they also had half or more of their alveolar and hard palate removed. ${ }^{5,21}$ 
The stage and type of tumor could also affect the QoL, the severity of patient's symptoms and their psychological distress. ${ }^{49,52,55-61}$ Regarding the tumor stage, several studies reported that QoL is lower in patients who have large tumors and late stage. ${ }^{58-61}$ In our search, only one study reported tumor stage, However, the data in the this study suggested that all approaches should be discussed with the patients without concern about the stage of tumors. ${ }^{52}$ Another factor that may affect the function of obturator prostheses and thus the patients' QoL is the adjuvant or postoperative radiotherapy, as xerostomia and severe trismus may create problems with impression procedures and difficulties with inserting, fitting and retention of obturators. ${ }^{49}$ Also radiotherapy may affect free tissue transfers resulting in impaired vascular function, poor healing conditions and tissues with low quality, this affecting the closure of the defect and the need for additional surgeries. ${ }^{57,62,63}$ Probably, patients subjected to such adjuvant treatment have tumors in advanced stages and may develop depression and physiologic distress. ${ }^{14}$ where in the included studies patients received adjuvant or postoperative radiotherapy were distributed equally in two studies. ${ }^{32,49}$ Thus, the age, the size of defects, the type of tumor, the stage of tumor and the adjuvant radiotherapy should be taken into consideration during the selection of treatment options.

The included studies are providing clinically relevant scenarios although they were derived from geographically diverse locations where exploring the impact of these parameters on different populations reflecting cultures regarding evaluation of both rehabilitations procedures. ${ }^{32,49-52}$

The obturator stability and retention could be significantly affected after an extensive resection due to insufficient remaining maxillary bone or teeth thus the advent of osseointegrated implants had significantly improved the obturator retention and stability. ${ }^{64-67}$ Also the presence of teeth or implants in surgically reconstructed area may play a role in the success of prosthetic rehabilitation of patients. ${ }^{45,49}$ Where in the included studies four studies were managed with installing implants but the importance of implants regarding the QOL were not evaluated. ${ }^{32,49-52}$

A direct comparison of the QoL scores among the selected studies was a challenge because nearly two studies employed the same questionnaire EORTC Head and Neck $35^{32,50}$ and evaluated functions of swallowing and speech. ${ }^{49,52}$ Additionally, the way in which the results were presented varied among the studies (Tab 3). It is worthy to mention that QoL researches showed that physical and emotional functioning and patients' overall health status are lowest at diagnosis and during the postsurgical period, where the main changes occurs in the first year after surgery. So, most variables return to pretreatment values. ${ }^{68-70}$ Additionally, patients try to adapt over time and underreport deficits. $^{71,72}$ Thus, in a longitudinal study starting before starting the treatment may show a larger differences between groups .

\section{Conclusions:-}

Surgical rehabilitation provide a better line of treatment in improving the QOL for patients with maxillary defects , On the other hand prosthetic has proved effectiveness in the immediate post-surgical times as temporary strategy, and it has represented a good alternative when the surgical obturation is compromised. Future studies should be prospectively designed describing in more detail the inclusion and exclusion criteria, evaluation QoL and best line of treatment. Consensus should be obtained regarding the standardization of the method for QoL evaluation, using validated questionnaires and validated objective tests, before and after oral rehabilitation, with adequate follow-up.

\section{References:-}

1. Glinski B, Zabek M, Urbanski J (2006) Principles of diagnosis and treatment of patients with head and neck squamous cancer. ContempOncol (Pozn) 6:263-267.

2. Yanamoto S, Yamada S, Takahashi H, et al(2014)Benefits of maxillectomy with internal dissection of the masticator space by transmandibular approach in the surgical management of malignant tumours of the upper gingiva and hard palate: A clinical review of 10 cases. Int J Oral MaxillofacSurg 11:1319-25

3. Nagy J, Braunitzer G, Antal M, et al (2014) QOL in head and neck cancer patients after tumor therapy and subsequent rehabilitation: An exploratory study. Qual Life Res 1:135-43.

4. Yucel A, Cinar C, Aydin Y, et al (2000) Malignant tumors requiring maxillectomy. JCraniofacSurg 11:418-29.

5. Brown JS, Rogers SN, McNally DN, Boyle M (2000) A modified classification for the maxillectomy defect. Head Neck 2000;22:17-26.

6. Baliarsing AS, Vinay VK, Neelima AM, Dilip KB (2010) Reconstruction of maxillectomy defects using deep circumflex iliac artery-based composite free flap. Oral Surg Oral Med Oral Pathol Oral RadiolEndod 109: 8-13. 
7. Cordeiro PG, Santamaria EA (2000)Classification system and algorithm forreconstruction of maxillectomy and midfacial defects. PlastReconstrSurg 105:2331-46.

8. 8- Futran ND, Mendez E (2006) Developments in reconstruction of midfaceandmaxilla. Lancet Oncol 7:24958.

9. Muzaffar AR, Adams Jr WP, Hatorg JM, Rohrich RJ, Byrd HS (1999) Maxillary reconstruction: functional and aesthetic considerations. PlastReconstSurg 104:2172-83.

10. Funk GF, Karnell LH, Christensen AJ (2012) Long-term health related quality of life in survivors of head and neck cacner. Arch Otolaryngol Head Neck Surg 138:123-33.

11. Zabora J, Brintzenhofeszoc K, Curbow B, Hooker C, Piantadosi S (2001) The prevalence of psychological distress by cancer site. Psychooncology 10:19-28.

12. Argerakis GP (1990) Psychosocial considerations of the post-treatment of head and neck cancer patients. Dent Clin North Am 34:285-305.

13. Hammerlid E, Persson L, Sulivan M, Westin T(1999) Quality-of-life effects of psychosocial intervention in patients with head and neck cancer. Otolaryngol Head Neck Surg 120:507-16.

14. Depprich R, Naujoks C, Lind D, Ommerborn M, Meyer U, Kübler NR, et al (2011) Evaluation of the quality of life of patients with maxillofacial defects after prosthodontic therapy with obturator prostheses. Int J Oral MaxillofacSurg 40:71-9.

15. Irish J, Sandhu N, Simpson C, Wood R, Gilbert R, Gullane P, et al(2009) Quality of life in patients with maxillectomy prostheses. Head Neck 31:813-21.

16. 16 -Rogers SN (2009) Quality of life for head and neck cancer patients-Has treatment planning altered? .Oral Oncol 45:435-39.

17. Dalgorf D, Higgins K (2008) Reconstruction of the midface and maxilla. CurrOpinOtolaryngol Head Neck Surg 16:303-11.

18. 18-Rieger JM, Tang JA, Wolfaardt J, Harris J, Seikaly H(2011) Comparison of speech and aesthetic outcomes in patients with maxillary reconstruction versus maxillary obturators after maxillectomy. J Otolaryngol Head Neck Surg 40:40-7.

19. Kornblith AB, Zlotolow IM, Gooen J, Huryn JM, Lerner T, Strong EW, et al(1996)Quality of life of maxillectomy patients using an obturator prosthesis. Head Neck 18:323-34.

20. Sharma AB, Beumer J III (2005) Reconstruction of maxillary defects: the case for prosthetic rehabilitation. J Oral MaxillofacSurg 63:1770-3.

21. Okay DJ, Genden E, Buchbinder D, Urken M (2001) Prosthodontic guidelines for surgical reconstruction of the maxilla: a classification system of defects.JProsthetDent 86:352-63.

22. Brown JS, Shaw RJ (2010) Reconstruction of the maxilla and midface: introducing a new classification. Lancet Oncol 11:1001-8.

23. Nguyen CT, Driscoll CF, Coletti DP (2011) Reconstruction of a maxillectomy patient with an osteocutaneous flap and implant-retained fixed dental prosthesis: a clinical report. J ProsthetDent 105:292-5.

24. Marx RE (2009) Reconstruction of defects caused by bisphosphonate-induced osteonecrosis of the jaws. J Oral MaxillofacSurg 67:107-19.

25. Ruggiero SL, Dodson TB, Fantasia J, Goodday R, Aghaloo T, Mehrotra B, et al (2014) American Association of Oral and Maxillofacial Surgeons position paper on medication-related osteonecrosis of the jawse2014 update. J Oral MaxillofacSurg 72:1938-56.

26. Chiapasco M, Abati S, RamundoG,Rossi A, Romeo E, Vogel G(2000) Behavior of implants in bone grafts or free flaps after tumor resection. Clin Oral Implants Res 11: 66-75.

27. Bodard AG, Bemer J, Gourmet R et al (2011)Dental implants and free fibula flap: 23 patients. Rev StomatolChirMaxillofac 112: 1-4.

28. Iizuka T, Hafliger J, Seto I, Rahal A, Mericske-Stern R, Smolka K (2005) Oral rehabilitation after mandibular reconstruction using an osteocutaneous fibula free flap with endosseous implants. Factors affecting the functional outcome in patients with oral cancer. Clin Oral Implants Res 16: 69-79.

29. WHOQOL. The World Health Organization Quality of Life assessment (WHOQOL): position paper from the World Health Organization. SocSci Med 1995; 41: 1403-1409.

30. WHOQOL. What quality of life? The WHOQOL Group. World Health Organization Quality of Life Assessment. World Health Forum 1996;17: 354-356.

31. Felce D, Perry J (1995) Quality of life: its definition and measurement. Res DevDisabil 16: 51-74.

32. Wang F, Huang W, Zhang C, Sun J, Qu X, Wu Y (2016) Functional outcome and quality of life after a maxillectomy: a comparison between an implant supported obturator and implant supported fixed prostheses in a free vascularized flap.Clin. Oral Impl. Res 00: 1-7. 
33. Miller SA, Forrest JL(2001) Enhancing your practice through evidence-based decision making: PICO, learning how to ask good questions. J Evid Base Dent Pract 1:136-41.

34. Moher D, Liberati A, Tetzlaff J, Altman DG (2010) PRISMA Group. Preferred reporting items for systematic reviews and meta-analyses: the PRISMA statement. Int J Surg 8:336-41.

35. Welch V, Petticrew M, Tugwell P, Moher D, O’Neill J, Waters E, et al(2012) PRISMA-Equity 2012 extension: reporting guidelines for systematic reviews with a focus on health equity. PLoS Med 9:1-7.

36. Viswanathan M, Ansari MT, Berkman ND, Chang S, Hartling L, McPheeters LM, Santaguida PL, Shamliyan T, Singh K, Tsertsvadze A, Treadwell JR(2012) Assessing the risk of bias of individual studies in systematic reviews of health care interventions. Agency for Healthcare Research and Quality Methods Guide for Comparative Effectiveness Reviews. March 2012. AHRQ Publication No. 12- EHC047-EF. Available at: http://www.effectivehealthcare IndividualStudies.pdf

37. Rieger J, Bohle Iii G, Huryn J, Tang JL, Harris J, Seikaly H (2009) Surgical reconstruction versus prosthetic obturation of extensive soft palate defects: a comparison of speech outcomes. Int J Prosthodont 22:566-72.

38. BorlaseG(2000) Use of obturators in rehabilitation of maxillectomy defects. Ann R AustralasColl Dent Surg 15:75-9.

39. Rieger JM, Wolfaardt JF, Jha N, Seikaly H (2003) Maxillary obturators: the relationship between patient satisfaction and speech outcome. Head Neck 25:895-903.

40. Moreno MA, Skoracki RJ, Hanna EY, Hanasono MM (2010) Microvascular free flap reconstruction versus palatal obturation for maxillectomy defects. Head Neck 32:860-8.

41. Lethaus B, Lie N, de Beer F, Kessler P, de Baat C, Verdonck HW(2010) Surgical and prosthetic reconsiderations in patients with maxillectomy. J Oral Rehabil 37:138-42.

42. Hertrampf K, Wenz HJ, Lehmann KM, Lorenz W, Koller M (2004) Quality of life of patients with maxillofacial defects after treatment for malignancy. Int J Prosthodont 17:657-65.

43. Riaz N, Warriach RA (2010) Quality of life in patients with obturator prostheses. J Ayub Med Coll Abbottabad 22:121-5.

44. Kreeft AM, Krap M, Wismeijer D, Speksnijder CM, Smeele LE, Bosch SD,et al(2012)Oral function after maxillectomy and reconstruction with an obturator.Int J Oral MaxillofacSurg 41:1387-92.

45. Chigurupati R, Aloor N, Salas R, Schmidt BL(2013) Quality of life after maxillectomy and prosthetic obturator rehabilitation. J Oral MaxillofacSurg 71:1471-8.

46. Kumar P, Alvi HA, Rao J, Singh BP, Jurel SK, Kumar L, et al(2013) Assessment of the quality of life in maxillectomy patients: A longitudinal study. J AdvProsthodont 5:29-35.

47. Said MM, Otomaru T, Yeerken Y, Taniguchi H(2017) Masticatory function and oral health-related quality of life in patients after partial maxillectomies with closed or open defects . J Prosthet Dent 118:108-112.

48. Costa H, Zenha H, Sequeira H, Coelho G, Gomes N, Pinto C, Martins J,Santos D, Andresen C (2015) Microsurgical Reconstruction Of The Maxilla - Algorithm And Concepts.British Journal of Plastic Surgery 68 :89-104

49. Genden EM, Okay D, Stepp MT, Rezaee RP, Mojica JS, Buchbinder D, et al (2003)Comparison of functional and quality-of-life outcomes in patients with and without palatomaxillary reconstruction: a preliminary report. Arch Otolaryngol Head Neck Surg 129:775-80.

50. Rogers SN, Lowe D, McNally D, Brown JS, Vaughan ED (2003) Health-related quality of life after maxillectomy: a comparison between prosthetic obturation and free flap. J Oral MaxillofacSurg 61:174-81.

51. Pompa G, Brauner E, Jamshir S, De Angelis F, Giardino R, Di Carlo S(2017) Quality of Life in Patients Rehabilitated with Palatal Obturator without Reconstruction Versus Fixed Implant-Prosthesis after Reconstruction of Maxillectomy Defects . J Int Dent Med Res 10:1-8.

52. Murphy J, Isaiah A, Wolf JS, Lubek JE(2015) Quality of life factors and survival after total or extended maxillectomy for sinonasal malignancies. J Oral MaxillofacSurg 73:759-63.

53. Brauner E, Valentini V, Jamshir S, Guarino G, Battisti A, Fadda MT, Pompa G (2016) Retrospective review of 78 rehabilitated head and neck postoncological patients: a new classification method. Minerva Stomatologica 65:17-38.

54. Australian Government, NHMRC. How to use the evidence: assessment and application of scientific evidence. Available at:http://www.nhmrc.gov.au/_files nhmrc/file/publications/synopses/cp69.pdf. Accessed April 24,2015.

55. Martín-Ares M, Barona-Dorado C, Guisado-Moya B, Martínez-Rodríguez N, Cortés-Bretón-Brinkmann J, Martínez-González JM(2015)Prosthetic hygiene and functional efficacy in completely edentulous patients: satisfaction and quality of life during a 5-year follow-up. Clin Oral Implants Res 20:1-6. 
56. Awad MA, Lund JP, Shapiro SH, Locker D, Klemetti E, Chehade A, et al (2003) Oral health status and treatment satisfaction with mandibular implant overdentures and conventional dentures: a randomized clinical trial in a senior population. Int J Prosthodont 16:390-6.

57. Dings JP, Mizbah K, Bergé SJ, Meijer GJ, Merkx MA, Borstlap WA (2014) Secondary closure of small- to medium-size palatal defects after ablative surgery: reappraisal of reconstructive techniques. J Oral MaxillofacSurg 72:2066-76.

58. Rana M, Kanatas A, Herzberg PY, Khoschdell M, KokemuellerH,Gellrich NC, et al (2015) Prospective study of the influence of psychological and medical factors on quality of life and severity of symptoms among patients with oral squamous cell carcinoma. Br J Oral MaxillofacSurg 53:364-70.

59. Gellrich NC, Schimming R, Schramm A, Schmalohr D, Bremerich A, Kugler J ( 2002) Pain, function, and psychologic outcome before, during, and after intraoral tumor resection. J Oral MaxillofacSurg 60:772-7.

60. Kollbrunner J, Zbären P, Quack K (2001) Quality of life stress in patients with larger tumors of the mouth. A descriptive study of psychosocial effects of illness and primary surgery therapy in 3 partsd1: Quantity and quality of life. HNO 49:985-97.

61. Aarstad AK, Beisland E, AarstadHJ( 2012) Personality, choice of coping and T stage predict level of distress in head and neck cancer patients during follow-up. Eur Arch Otorhinolaryngol 269:2121-8.

62. van der Sloot PG (2003) Hard and soft palate reconstruction. CurrOpinOtolaryngol Head Neck Surg 11:225-9.

63. Batchelor AG, Palmer JH (1990) A novel method of closing a palatal fistula: the free fascial flap. Br J PlastSurg 43:359-61.

64. Boyes-Varley J.G, Howes D.G, Davidge-Pitts K.D, Branemark I, McAlpine J.A(2007) A protocol for maxillary reconstruction following oncology resection using zygomatic implants. The International Journal of Prosthodontics 20:521-531.

65. Hu Y.J, Hardianto A, Li S.Y, Zhang Z.Y, Zhang C.P (2007) Reconstruction of a palatomaxillary defect with vascularized iliac bone combined with a superficial inferior epigastric artery flap and zygomatic implants as anchorage.International Journal of Oral Maxillofacial Surgery 36: 854-857.

66. Weischer T, Schettler D, Mohr C (1997)Titanium implants in the zygoma as retaining elements after hemimaxillectomy. International Journal of Oral and Maxillofacical Implants 12: 211-214.

67. Leles C.R, Leles J.L, de Paula Souza C, Martins R.R , Mendonc E.F(2010) Implant-supported obturatoroverdenture for extensive maxillary resection patient: a clinical report. Journal of Prosthodontics 19: 240-244.

68. Schliephake H, Jamil MU (2002) Prospective evaluation of quality of life after oncologic surgery for oral cancer. Int J Oral MaxillofacSurg 31: 427-33.

69. Shepherd KL, Fisher SE (2004) Prospective evaluation of quality of life in patients with oral and oropharyngeal cancer: from diagnosis to three months post-treatment. Oral Oncol 40: 751-7.

70. Rogers SN, Lowe D, Humphris G (2000) Distinct patient groups in oral cancer:a prospective study of perceived health status following primary surgery. Oral Oncol 36:529-38.

71. Allison PJ, Locker D, FeineJS(1997) Quality of life: a dynamic construct. SocSciMed ;45:221-30.

72. Breetvelt IS, Van Dam FS(1991) Under reporting by cancer patients: the case of response-shift. SocSci Med 1991;32:981-7. 